\title{
СХОДИМОСТЬ И УСТОЙЧИВОСТЬ РАЗНОСТНЫХ СХЕМ ДЛЯ РЕЩІЕИЯ НЕЛИНЕЙНЫХ ЭВОЛЮЦИОННЫХ УРАВНЕНИЙ
}

\author{
๑. ๑. HBAHAYCKAC
}

Department of Mathematics, Vilnius University

Naugarduko 24, Vilnius 2006, Lithuania

E-mail: feliksas ivanauskas maf. nu. It

1. Веедешие. Работа посвящена обоснованию разностных методов для решения нелинейных эволюционных уравнений (НЭУ). Основное внимание уделено нелинейнњ уравнениям Шрединтера (НУШ). Результаты работы также перенесены на уравнения Курамото-Цузуки (КЦ) и уравнения типа реакция-диффузия (РД). НУШ описывағот многие задачи нелиненно оптики $[1,2]$. Они такхе используются в физике плазмы, квантовой физике, молекулярной биологии. Уравнение КЩ описывает поведение многих двухкомпонентных систем в окрестности точки бифуркации [3]. Системы типа РД применяют при исследовании широкого класса нелинейных процессов [4].

Работа является обобщением и развитием резуљьтатов [5-7]. Основнымя моментами обоснования разностных методов для решения НЭУ являются:

a) примененне нетрадиционных априорных оценок,

б) использование мультипликативнњх оценок типа Гапярдо-Ниренберга,

в) введение сеточных норм, учитывающих спектральные свойства линейного уравнения Шрединтера (ЛУШ).

Исследование разностных методов для решения НУШ по сравнению с уравнениями параболипеского типа стапкиваются с двумя трудностями, которые обнаружяваются уже на уровне линейных уравненић. Во первых, для ЛУШ не имеет места принцип максимума и отсутствуют соответствующщие априорные оценки. Во вторқх, для устойчивости явной разностной схемы для ЛУШ возникает одень хесткое и неетественное условие на соотношение мехду шагами сетки $\tau / h^{4} \leq c$. Омметим, тто безразмерной величиной является отношение $\tau / h^{2}$, а не $\tau / h^{4}$ [8].

Дия решгения НУШ, как правило, имеют место априорные оценки в классе $L_{2}$, в некоторых случаях в $W_{2}^{1}$. Поэтому при исследовании разностных схем для НУШ была предпринята попытка обобщить понятие ктассичесхих априорных оценок и получять эффективные оценки. В классических априорных оценках

$$
\|u(t)\| \leq c \mid u(0) \|
$$

постоянная $\mathcal{C}$ не зввисит от решения дифференщиальной задачи. В работах [5-7] введено обобщение априорньгх оценок вида

$$
\|\boldsymbol{u}(\boldsymbol{t})\|_{W_{2}^{\prime}} \leq c \mid\|\boldsymbol{L}(0)\|_{W_{2}^{\prime}},
$$

где постоянная $c$ уже зависит от некоторой нормы решения дифференщиащьой задачи и ллины отрезка изменения эволюционной переменной. Так для $n+1$-мерной $(\boldsymbol{n}$ - тисло пространсгвеннhх переменных) задачи постоянная $c$ зависит от нормы решения $C^{j}(Q)$, где $\left.Q=10, T^{\prime}\right] \times \bar{\Omega}$, $j=[(n-1) / 2]$.

При исследовании сходимости разностньх методов ция решгения НУШ актуағьно наличие априорных оценок в норме $C$. Такая оценка сводит вопрос о сходимости нелиненной разностной схемы по сушеству к вопросу об исследовании линейной разностной схемы. Так как для решения лУШ имеют место априорғые оценки в норме $W_{2}^{l}$, по возникла идея о получении априорных оце- 
нок в норме $W_{2}^{l}$ для решгения разностной задачи. Для этого при исследовании НУШ были получены обобщенные классические априорные оценки вида

$$
\|\boldsymbol{L}(t)\|_{W_{2}^{k}} \leq c\|\boldsymbol{L}(0)\|_{W_{2}^{d}},
$$

где постояная $c$ ухе зависит от нормы решения дифференциатной задачи в $C^{j}(Q)$ и дшины отрезка изменения эволюционно пе переменно оц. Оценки этого вида были получены как для дифференциальой, так и для разностной задач. В случае дифференщиальной задати такая оценка дает каqественную характеристику решения. Наиболыней ценностыо априорных оценок вида (1.1) является то, что в случае сеточных норм постоянную $c$ удается освободить от зависимости нормы решения разностной задачи $C^{j}\left(Q_{h}\right)$. Точнее, норму решения разностной задачи удается заменить через норму решения исходно дифференцияльной задачи. В итоге получяем эффективную априорную оценку для решения разностной задачи в норме $W_{2}^{l}$. Эта априорная оценка играет вахную рољь при доказателстве сходимости и устойчивости разностной схемы.

В численном анализе при исследовании нелинен̆нб разностных схем традиционно применяется оценка

$$
\|u\|_{C} \leq c h^{-n / 2}\|u\|_{L_{2}}
$$

где $\boldsymbol{n}$-число измерений. Оценка (1.2) является типичной оценкой численного анализа и не имеет непрерывного аналога. Использование это оценки при обосновании разностных схем приводит, как правило, $\mathbf{k}$ огранндению на соотношение мехду шагами по эволюиионно й и пространственным переменғым. Следует отметить, что при помощи оценпи (1.2) и двухсторонних оценок мохно получить [9] такхе безусловную сходимость при $n=2$. Однахо, при $n>2$ использование оценки (1.2) становится не только малооффективным, но чясто невозмохным. В работах [5-7] вместо оценки (1.2) было предлохено испольовать мультиликативные оценки типа Галярдо-Ниренберга

$$
\|u\|_{C} \leq c\left|u\left\|_{L_{2}}^{\alpha}\right\| u\right|_{W_{2}^{d}}^{1-\alpha}
$$

Применение этих оценок требует гладкости решения разностной задачи по крайней мере в норме $W_{2}^{l}, 2 l(1-\alpha)>1,1>\alpha>(n-1) / n$. Эо гарантирует ограниченность решения в норме $C$. Такая глалкость решения в норме $W_{2}^{l}$ согласуетея в нехоторой степени с глапкостью решения дифференциаљьно задачи необхомо дия аппроксимации. Поэтому естественно охидать, что решение разностной задачи в сеточно н норме мохет иметь ту хе глапкость решеения дифференциањной задачи. Почему выбрана норма $W_{2}^{l}$ ? Этот выбор обусловлен наличием априорнбх оценок для ЛУШ в $W_{2}^{l}$ и спектрапьными свойствами ЛУШ

$$
\frac{\partial u}{\partial t}=i \Delta u, \quad i^{2}=-1,
$$

которое имеет тригонометрические функции в качестве собственњых. В случае равномерной сетки тригонометрические функции (синусы и косинусы) обладают ортогонаљьостъю в соответственно подобранных сеточных нормах. Поэтому сетопную норму мохно определитъ, испољьуя равенство Парсеваля. Это позволяет полуучитъ сетопнын аналог априорной оценки разностной задачи для ЈУШ в норме $W_{2}^{l}$, что в конечном итоге позволяет получитъ зффективные априорные оцекпи для решгения разностной задачи в норме $W_{2}^{l}$. Следует также отметить, что разрешимость нелинейной разностной задачи, сходимость и устойчивость схемы в суиности являются следствием предполохения о существовании достаточно гладкого решения НУШ. Последнее суждение можно сформулировать еше и так: если существует достаточно гладкое решение НУШ, то можно построить разностную схему, решение которой сходится, и еще имеют место оценки устойивости решения разностной задачи. Это высказывание как бы является дапьнейшим обобщением основополагаюшей теоремы разностных методов о том, что из аппроксимации и устойчивости вытекает сходимостъ. В данном случае условие устойчивости гарантируется обобщенными априорными оценками для 
НУШ. В работе исследуются разностные схемы типа Кранка-Никольсона для решения первой и второй краевых задач и задачи Коши пाя нелинейныг эволюционньх уравнени

2. Сходамость. Устонтиость. Пусть $\Omega=\left\{x .0<x_{i}<1, i=1, \ldots, n\right\}$ есть $n$-мернын единияный куб в $R^{n}, \partial \Omega$-граница области и $\left.\left.Q=\right] 0, T\right] \times \Omega$. Основные результаты излохим на примере второй краевоћ задачж для системы НУШ

$$
\begin{aligned}
& \frac{\partial u}{\partial t}=\sum_{j=1}^{n} A_{j} \frac{\partial^{2} u}{\partial x_{j}^{2}}+F(u), \quad(t, x) \in Q . \\
& u(0, x)=u^{(0)}(x), \quad x \in \bar{\Omega}, \\
& \left.\left.\frac{\partial u(t, x)}{\partial n}=0, \quad(t, x) \in\right], T\right] \times \partial \Omega .
\end{aligned}
$$

Здесь $A_{j}$-диагональные метрицы с мнимыми коэффициентами. Если дияананњные матрицы $A_{j}$ с комплексными коэффициентами, то (2.1) является уравнениями КЦ, если хе с действитељныди коэффициентами, то (2.1) является системой уравнений типа РД. Отметим, что исследованде системы (2.1) ввиду днагонашьости матриц $A_{j}$ приншипиально не отличается от исследования одного уравнения. Поэтому в дапьненем будем рассмятривать одно уравнение

$$
\begin{aligned}
& \frac{\partial u}{\partial t}=L u+f(u), \quad(t, x) \in Q, \\
& u(0, x)=u^{(0)}(x), \quad x \in \bar{\Omega}, \\
& \left.\left.\frac{\partial u(t, x)}{\partial n}=0, \quad(t, x) \in\right] 0, T\right] \times \partial \Omega, \\
& L u=\sum_{j=1}^{n} a_{j} \frac{\partial^{2} u}{\partial x_{j}^{2}},
\end{aligned}
$$

где $a_{j}$-комплексые числа

В болынинстве задач [1-4] функиия $f(u)$ является многочленом. Поэтому в даљнейшем ограничимся стучаем фунции $f(u)$, явяяощеися многочленом. Кроме того, будем придерживатъся обозначенин [8].

Задаяе (2.4)-(2.6) поставим в соответствие неявную схему с весами Кранха-Никольсона

$$
\begin{aligned}
& p_{t}=L_{h} p^{(\sigma)}+g(p, \hat{p}), \quad(t, x) \in Q_{h}, \\
& p(0, x)=u^{(0)}(x), \quad x \in \bar{\Omega}_{h}, \\
& l_{h} p^{(\sigma)}=0, \quad(t, x) \in \omega_{\tau} \times \partial \Omega_{h},
\end{aligned}
$$

где

$$
\begin{aligned}
& L_{h} p=\sum_{j=1}^{n} a_{j} p_{\bar{x}_{j} x_{j}}, \\
& l_{h} p^{(\sigma)}=p_{x_{k}}^{(\sigma)}-\frac{h}{2 a_{k}}\left(p_{t}-\sum_{j=1, j \neq k}^{n} a_{j} p_{\bar{x}_{j} x_{j}}^{(\sigma)}-g(p, \hat{p})\right)
\end{aligned}
$$

Предполохим, что

\section{a) $0.5 \leq \sigma \leq 1$}

Функция $g(p, \hat{p})$ является многочленом относительно $p, \hat{p}$. В простеншем случае можно взять $g(p, \hat{p})=f\left(p^{\sigma}\right)$. Сформулируем утверждение, дагшее основу для сушествования априорной оценки решения разностной задачи. 
Теорема 1. Пусть выполкено условие а) и сущестөует решение зодачи (2.4)-(2.6)

$$
p \in W_{2}^{l}\left(\Omega_{h}\right) \cap C^{j}\left(Q_{h}\right), \quad j=[(n-1) / 2] .
$$

Тогда существует такая постоянная $\tau_{0} \geq 0$, ито при $\tau \leq \tau_{0}$ справедлива оченка

$2 \partial e$

$$
\|p(t)\|_{W_{2}^{\prime}\left(\Omega_{h}\right)} \leq c\|p(0)\|_{W_{2}^{\prime}\left(\Omega_{h}\right)}, \quad t \in \bar{\omega}_{\tau}
$$

$$
c=c\left(\|p\|_{C^{J}\left(Q_{h}\right)},\|p(0)\|_{W_{2}^{l-1}\left(\Omega_{h}\right)}, T, n, l\right) .
$$

Сформулируем утверхдение о сходимости разностной задачи.

Теорема 2. Пусть выполнено условие а) и $r=2(l-s)(1-\alpha)-1>0, \quad 1>\alpha>(n-1) / n$. Тогда суиестеуюот такие постоянные $\tau_{0}, h_{0}>0$, ито при $\tau \leq \tau_{0}$, $h \leq h_{0}$ сунестөует единственкое решекие задачи (2.7)-(2.9), соодящееск к решению задачи (2.4)-(2.6) и справедливы оченки

$$
\begin{aligned}
& \|\boldsymbol{u}(t)-p(t)\|_{W_{2}^{s}\left(\Omega_{h}\right)} \leq c \max _{\omega_{\gamma}}\|\Phi(t)\|_{W_{2}^{s}\left(\Omega_{h}\right)}, \\
& \|\mathcal{L}(t)-p(t)\|_{C_{2}\left(\Omega_{h}\right)} \leq c\left(\max \mid \Phi(t) \|_{\omega_{7} W_{2}^{s}\left(\Omega_{h}\right)}\right)^{\beta},
\end{aligned}
$$

$t \in \omega_{\tau}, \quad s \leq l-n \backslash 2, \quad \beta=r /(r+1)$, zде $\Phi(t)$-погрешность аппроксимачии.

Исследуем устойчвость. Пусть $u_{1}, u_{2}$ и $p_{1}, p_{2}$-решения задаq $(2.4)-(2.6)$ и (2.7)-(2.9) с начальными данными $u_{1}^{(0)}, u_{2}^{(0)}$. Справедлива

Теорема 3. Пусть выполнены условия теоремы 1. Тогда суцествуют такие постояниве $\tau_{0}, h_{0}>0$, uто при $\tau \leq \tau_{0}, h \leq h_{0}$ справедливо оченко

ade nостояниая $c$ не зависит от $\tau, \hat{h}$.

$$
\left\|p_{1}(t)-p_{2}(t)\right\|_{L_{2}\left(\Omega_{h}\right)} \leq c\left\|u_{1}(0)-u_{2}(0)\right\|_{L_{2}\left(\Omega_{h}\right)}, \quad t \in \omega_{\tau}
$$

Эти резулттаты переносятся на уравнения КЦ и РД. В работе [10], применяя эту технику, обоснована схема расщепления.

\section{Лrтepatypa}

1. А П. Сухоруков. Нелинейное волновое взаимодействие в оптике и радиофизике, М.: Наука, 1988.

2. Ю. Н. Карамзин, А П. Сухоруков, В. А Трофимов. Математическое моделирование в нелинейной оптике, М.: Изд-во Моск. ун-та, 1989.

3. А Ю. Лоскутов, А С. Михайлов. Введение в синергетику, М.: Наука, 1990.

4. C. V. Pao. Nonlinear Parabole and Eliptic Equations. Plenum Press, New York, 1992.

5. Ф. Ф. Иванаускас. Сходимось и устойтивость разностьљох схем для нелинейных уравнений Шрединтера, уравнения Курамото-Цузука и сястем типа реахция-диффузия. ДАН России, Т. 337 (1994), №5.

6. Ф. Ф. Иванаускас. Сходимость и устойнивость разностных схем для нелинейных уравнений Шредингера, уравнения Курамото-Цузуки и систем типа реакция-диффузия. Лит. мат. сб. Т. 34 (1994), №1, c. $32-51$.

7. $\Phi$. $\Phi$. Иванаускас. Сходимость и устойчивость разностных схем для нелиненньбх уравнени шрединтеровского типа Лит. мат. с6., Т. 31 (1991), №4,с. 606-621.

8. A А Самарскин. Теория разностных схем, М.: Наукя, 1989.

9. А Б. Борисов. Симметрические схемы типа Кранка-Никольсона для решения нелинейных уравнений Шредингера. Методы и алгоритмы численного анализа и их приложения. Под редакцией Н. С. Бахвалова, В. А Морозова. Изд-во Моск. ун-та, 1989, с. 76-103.

10. Ф. Ф. Иванаускас. Метод расцепления пия решения нелинейньг уравнений шредингеровского типа, Ж. вычисл. матем. и физ. ,Т. 29 (1989), № 12,с. 1830-1838. 